\title{
BÚSQUEDA DE INFORMACIÓN Y REDES SOCIALES: EL CASO DE LA UNIVERSIDAD
}

Joan Francesc Fondevila-Gascón': Universitat Abat Oliba. España jfondevilag@uao.es

José Luis del Olmo-Arriaga: Universitat Abat Oliba. España jlolmo@uao.es

Javier Sierra Sánchez: Universitat Abat Oliba. España jsierras@uao.es

\section{RESUMEN}

Las redes sociales están convirtiéndose en un actor principal en el proceso de búsqueda de información. La elección de Universidad es uno de los procesos de decisión que están siendo condicionados por el fenómeno de las redes sociales. Como reflejo del carácter conversacional y descentralizado de Internet, el universo 2.0, aprovechando las ventajas de la Sociedad de la Banda Ancha, se está constituyendo para los estudiantes como una de las principales vías de información. Las Universidades comienzan a percatarse de ello, e intentan adaptar sus estrategias de marketing para captar al alumnado. En este artículo analizamos el papel de las redes sociales como fuente de información para los estudiantes que pretenden acceder a la Universidad, y las comparamos con otros medios de comunicación.

PALABRAS CLAVE: Información - Redes sociales - Universidad - Internet - Banda Ancha.

\footnotetext{
${ }^{1}$ Autor correspondiente

Joan Francesc Fondevila-Gascón: Profesor de Tecnología, Profesor del Doctorado en Sociedad de la Información y el Conocimiento y director del CECABLE (Centro de Estudios sobre el Cable). Universitat Abat Oliba CEU (UAO), Universitat Oberta de Catalunya (UOC) y CECABLE. Barcelona (España).

Correo: jfondevilag@uao.es
} 


\title{
INFORMATION RESEARCH AND SOCIAL NETWORKS: THE CASE OF THE UNIVERSITY
}

\begin{abstract}
The social networks are turning into a principal actor in the process of information search. The choice of University is one of the processes of decision determined by the phenomenon of the social networks. As reflection of the conversational and decentralized character of the Internet, the universe 2.0, taking advantage of the Broadband Society, is constituted for the students as one of the principal routes of information. The Universities begin to notice it, and try to adapt their strategies of marketing to catch to the student body. In this article we analyze the paper of the social networks as source of information for the students who try to accede to the University, and compare them with other mass media.
\end{abstract}

KEY WORDS: Information - Social Networks - University - Internet - Broadband

\section{INTRODUCCIÓN}

Las redes sociales están convirtiéndose paulatinamente en uno de los factores angulares en la toma de decisión estudiantil sobre la elección de Universidad. El fenómeno, cuyo alcance cuantitativo es innegable, está alertando a los gestores encargados de la captación de estudiantes en las Universidades. Las estrategias de marketing se adaptan y se perfeccionan con ese fin, por cuanto las redes 2.0 son una fuente de información creciente en el momento de acceder a la Universidad. Establecer una comparativa con otros medios de comunicación es clave para ponderar el impacto actual de las redes sociales.

\subsection{El creciente impacto de las redes sociales y el web 2.0 en la búsqueda de información y en la Universidad}

\section{La introducción de las redes sociales en la praxis de la Universidad}

La educación superior, en su empeño por mejorar fórmulas de captación de alumnado y también vías pedagógicas en la implementación de la docencia, comienza a percibir el fenómeno web 2.0, encabezado por las redes sociales, como una oportunidad a explorar. En determinados tipos de estudios universitarios, sobre todo los centrados en la creación de contenidos (encabezados por el Periodismo) y los 
Bolonia invita a combinar sistemas de evaluación muy diversos: pruebas objetivas, pruebas de respuestas cortas, pruebas de desarrollo, disertaciones, participación activa del alumno, ensayos o informes, proyectos, pruebas de ejecución de tareas, técnicas de observación, portafolio, memorias de prácticas, sistemas de autoevaluación, defensas de trabajos o exámenes orales, entre otras opciones.

Las metodologías docentes combinan una pléyade de fórmulas, algunas de ellas innovadoras, como el método expositivo, el estudio de caso, la resolución de ejercicios y problemas, el diseño de proyectos, el estado de la cuestión, el aprendizaje cooperativo, el trabajo de síntesis o el contrato de aprendizaje autónomo. Las actividades formativas suman, a la clásica lección magistral, fórmulas como el seminario, el taller, las prácticas internas o externas, la tutoría, el trabajo en grupo y el trabajo autónomo.

Introducir en ese elenco de opciones las redes sociales como fuente de actividades comienza a vislumbrarse como una obligación, sobre todo en la dinámica de aproximación de los contenidos universitarios al modus operandi y al ecosistema cada vez más habitual de los estudiantes: el digital. La práctica docente mejora empíricamente si sigue ese precepto (Fondevila Gascón y Carreras Alcalde, 2010).

Si la incorporación de las redes sociales en el ejercicio de la docencia parece inevitable, y comienza a ser analizada científicamente, el impacto de las redes sociales como factor de elección de Universidad apenas cuenta con estudios ni en España ni en el ámbito internacional, constatación sorprendente, ya que la cadena de desencadenantes de la elección de Universidad se hallan en la base de la previsión económica de las instituciones de educación superior. Sin un conocimiento fidedigno del impacto del universo 2.0, las estrategias de marketing de una Universidad pueden naufragar.

Cuando se analiza el proceso de toma de decisión de los estudiantes, los medios de comunicación son esenciales. Considerando a las redes sociales como un nuevo ecosistema comunicativo en empinado crecimiento, es imprescindible estudiarlas. El proceso de toma de decisiones combina una fase implícita (los consumidores pueden llegar a elegir posibles resultados) y una explícita o activa, en la que se decide sobre los productos a adquirir o consumir.

Los factores de elección de Universidad implican conocer cómo eligen los estudiantes entre las diferentes alternativas a las que pueden acceder, qué fórmulas utilizan para buscar la información sobre las diferentes alternativas, cuál es el grado de influencia 
A la hora de determinar cómo se toma la decisión, se produce una conjunción de condicionantes, como los mass media, el acceso a la información, la anticipación, la negociación familiar, el cálculo o la valoración (Veleda, 2002), aunque el proceso de elección depende sobre todo de los recursos económicos, culturales y sociales disponibles en las familias (Crozier, 1997).

Los medios de comunicación tradicionales y digitales, las visitas a actos diversos ${ }^{2}$ y las referencias ${ }^{3}$ son los tres focos de obtención de información analizados en este estudio. En este sentido, el salto de los centros de Secundaria a la Universidad requiere una correcta distribución de la información: el papel de las redes sociales y de los medios de comunicación es en este punto imprescindible.

La hipótesis de esta investigación es que consideramos a las redes sociales como una fuente de información relevante para los estudiantes que desean obtener datos sobre las Universidades. No obstante, las redes sociales y el resto de medios no permiten una valoración exhaustiva de las diferentes alternativas, y las referencias familiares gobiernan como fuente ante la información de los medios de comunicación y de las visitas.

\section{Búsqueda de información: el impacto de las redes sociales en los futuros estudiantes}

La consolidación de la Sociedad de la Banda Ancha (Fondevila Gascón, 2008) ha impulsado un uso intensivo y exponencial de Internet (Gilder, 2002), primero en una vertiente pasiva de descarga de información, más adelante como foco activo de incorporación de comentarios y contenidos de los internautas.

El imparable proceso de digitalización y de convergencia tecnológica multiplataforma y la expansión de las redes de banda ancha (Fondevila Gascón, 2011) colaboran en el crecimiento de la cantidad de usuarios de las redes sociales. Datos como que el 59,1\% de hogares españoles dispongan de acceso a Internet (Ontsi, 2011), y que el $64,2 \%$ de españoles se considere población internauta (las generaciones directamente impactadas por nuestra investigación lideran esa clasificación) reflejan el peso creciente de las redes sociales. A ellas se suman los medios de comunicación digitales.

Los usuarios de las nuevas herramientas sociales 2.0 se incrementaron del $45 \%$ al 81\% (The Cocktail Analysis, 2010). La cifra media de redes sociales a las que

${ }^{2}$ Los actos tenidos en cuenta en la investigación son los siguientes: ferias, jornadas, visitas personales 
pertenecía un usuario evolucionó de 1,7 en 2008 a 2,3 en 2009: el 64\% de usuarios utilizaba Facebook (un 13\% en 2008), y el 33\% Tuenti (el 12\% en 2008), la red social que generaba más engagement con los usuarios y la que encabezaba la frecuencia de acceso.

De hecho, el 75\% de usuarios accedían diariamente a Tuenti, que aventajaba en ese parámetro a Messenger (la herramienta de comunicación más utilizada, con el 68\%) y Facebook. Sea como fuere, el $80 \%$ de adictos accedían varias veces por semana a esas páginas web. Flickr y Twitter (10\% de usuarios) se conformaban con posiciones más rezagadas. En cuanto a la pérdida de usuarios o tasa de churn ${ }^{4}$, Fotolog, Hi5 y MySpace eran los principales perjudicados. El hecho de que el $45 \%$ de las cuentas abiertas en redes sociales no fuesen utilizadas alertaba de un cierto sobredimensionamiento del fenómeno. Los foros contaban con la participación del $27 \%$ de usuarios, aunque la tendencia era in diminuendo.

Los blogs cedían centralidad en porcentaje de penetración (15\%), pese a que mantenían una posición angular en lo referente a la difusión de contenidos y de conversaciones. Los datos estadísticos denotan, por tanto, que Internet es una referencia informativa clave (Ontsi, 2010). Así, en España, el 40\% de individuos utilizaban Internet para encontrar información sobre bienes y servicios; el 38\% para leer periódicos o revistas en línea; y el 29\% para buscar información relativa a la formación. El uso de Internet para interactuar con las autoridades públicas se situaba en el 30\%. Ello refleja que los pasos dados en España para desarrollar la eadministration aún deben agrandarse.

Observamos, pues, que, para los futuros estudiantes universitarios, fuentes de captación de información cada vez más atractivas son las páginas web de las Universidades, los portales de Internet especializados en información sobre educación superior, los diarios digitales (sean con referente en papel, sean pure players), los blogs y las redes sociales. Factores coadyuvantes son la navegabilidad y la comodidad de acceso a los contenidos digitales, amén de la inclusión acertada de elementos multimedia, interactivos e hipertextuales.

Esa oleada hacia las redes sociales conlleva un grado de exigencia mayor en los estudiantes, cuya actitud de prueba y exploración de esas ventanas virtuales comporta más disposición al cambio. 
Pese a ello, se pueden determinar perfiles de usuarios en función de su vivencia y hábitos en las redes sociales. Los Social Media Selectors (40\%) prueban diversas redes y se acaban decidiendo por una de ellas, buscan la funcionalidad comunicacional y el contacto con el grupo de referencia. Para los Simple Social Networks (33\%), la frecuencia de uso 2.0 (foros, blogs, redes sociales y correo electrónico) es menor, polarizada por redes universales, sobre todo Facebook y Tuenti, en una dimensión lúdica y relacional respecto a su grupo.

El de los Trend Followers (19\%) es el perfil más joven (16-25 años), muy activo, especialmente en Tuenti, Youtube y Fotolog, a la búsqueda de entretenimiento y de mantenerse informado sobre eventos o fiestas. Los Social Media Addicts (10\%) son minoritarios pero tendenciales. Más habituales en el segmento de 26 a 35 años, utilizan intensivamente las diferentes plataformas (mensajería instantánea, sobre todo Messenger y, menos, Skype, redes sociales, foros y blogs), diversifican tipologías de redes (exposición, microblogging 5 o profesionales), promueven la pluralidad y actúan con intencionalidad profesional.

El estudio sobre impacto de las redes sociales en la elección de Universidad parte de un territorio casi virgen. De hecho, los análisis sobre fuentes de información y correlación con la elección de Universidad son escasos en España, focalizados en variables concretas como el sexo (Frotuny et altri, 1991) o el rendimiento académico en el tránsito de secundaria a la Universidad (Fita et altri, 2004). El establecimiento de los motivos de elección (Porto y Mosterio, 2000) se revela complejo.

El intento de crear una herramienta de medida para estandarizar el proceso de elección de Universidad en función de dieciséis características o atributos del centro de enseñanza superior y la calidad (Roszkowski y Spreat, 2010) refleja la demanda en este sentido. El estudio de la imagen de la Universidad para atraer nuevos inscritos y el impacto en el proceso de decisión lleva a concluir que las características institucionales son prioritarias ante los recursos interpersonales o de información utilizados por los estudiantes (Pampaloni, 2010). Algunos autores han indicado como elementos decisionales la cercanía de la Universidad, la apariencia y el estado de las instalaciones (West et altri, 1991), pero, como se puede comprobar, analizar el impacto de los medios de comunicación es un reto científico a efectos de generar recursos para perfeccionar las estrategias de marketing.

Uno de los denominadores comunes que brota del marco teórico analizado es que la elección de Universidad se acostumbra a llevar a cabo en condiciones no idóneas. Las causas son diversas: desde la carencia de información hasta la ausencia de servicios de orientación educativa, pasando por condicionantes para ofertas de estudios en el

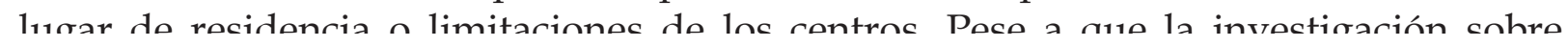


Unidos, Reino Unido, Canadá, Australia o Nueva Zelanda, a raíz de la implementación de políticas promotoras de la competencia interinstitucional para elegir centro, lo que afecta tanto a la educación superior como a la básica.

El objeto de estudio que analizamos adquiere más relevancia por la ascendente competencia interuniversitaria que se diagnostica en España y por la aparición de análisis comparativos. El escenario español es, en general, de carácter bipolar: unas Universidades públicas en las que, en determinadas titulaciones, se detecta una demanda superior a la oferta, y unas privadas en las que la oferta acostumbra a desbordar a la demanda. Ante esa realidad, la senda a seguir por las estrategias difiere. Se abre un abanico de amenazas y de oportunidades, y ahí las redes sociales se erigen en ventaja competitiva para la Universidad que las aplique antes y más intensivamente. Ello rompería la tradicional carencia de visión comercial en la gestión universitaria en España, causada por la sobredemanda pública. La ausencia de estudios empíricos que analicen el impacto de las redes sociales y páginas web en la elección de Universidad confiere a esta investigación un valor añadido.

\section{METODOLOGÍA}

\subsection{Uso de las redes sociales para elegir Universidad}

\section{Metodología para determinar el peso de las redes sociales en la elección de Universidad}

El análisis del uso de las redes sociales en el proceso de elección de Universidad se realizó mediante una encuesta diseñada por el Grupo de Investigación de la Universitat Abat Oliba CEU (UAO), en Barcelona (Cataluña), y llevada a cabo en noviembre de 2010. La muestra estaba compuesta por alumnos de primer curso de los Grados en Periodismo, Publicidad y Relaciones Públicas, Psicología, Derecho, Dirección de Empresas, Marketing y Dirección Comercial, Magisterio de Educación Infantil y Magisterio de Educación Primaria de la UAO.

El objetivo del trabajo cuantitativo consistía en conseguir datos que permitieran analizar el peso de las redes sociales como factor de búsqueda de información por parte de los estudiantes.

Las redes sociales se incluían entre diversas opciones, dentro del hiperónimo de medios de comunicación: anuncios en prensa, anuncios en radio, anuncios en televisión, anuncios en otros medios, página web de las Universidades, redes sociales 
El objetivo a largo plazo del Grupo de Investigación de la Universitat Abat Oliba CEU (UAO) es establecer oleadas de recogidas de datos que permitan un análisis longitudinal, lo que puede confirmar el peso efervescente de las redes sociales como epicentro de consultas para elegir Universidad. De hecho, las redes sociales comienzan a desbancar a medios de comunicación tradicionales.

\section{ANÁLISIS Y DISCUSIÓN}

\subsection{Datos empíricos sobre el peso de las redes sociales en la elección de Universidad}

Los resultados de la encuesta reflejan el peso de las redes sociales como fuente informativa para los estudiantes que pretenden acceder a la Universidad (Figura 1). Una cantidad que se aproxima al 9\% (el 8,98\%) de los encuestados buscó información a través de las redes sociales. Sólo las páginas web de las Universidades y las publicaciones de las Universidades superaban a las redes 2.0, lo que resulta significativo teniendo en cuenta el carácter relativamente neófito del ecosistema social virtual y su tendencia creciente, lo que le augura más posibilidades en todos los sentidos. Lo más natural es que en próximas oleadas el porcentaje de acceso a redes sociales como fuente de datos para decantarse por una Universidad o por otra crezca. La mejora en parámetros como el porcentaje de acceso a Internet, la fortaleza y expansión de las redes de banda ancha y el aumento de su caudal se combinan como estimulantes del fenómeno.

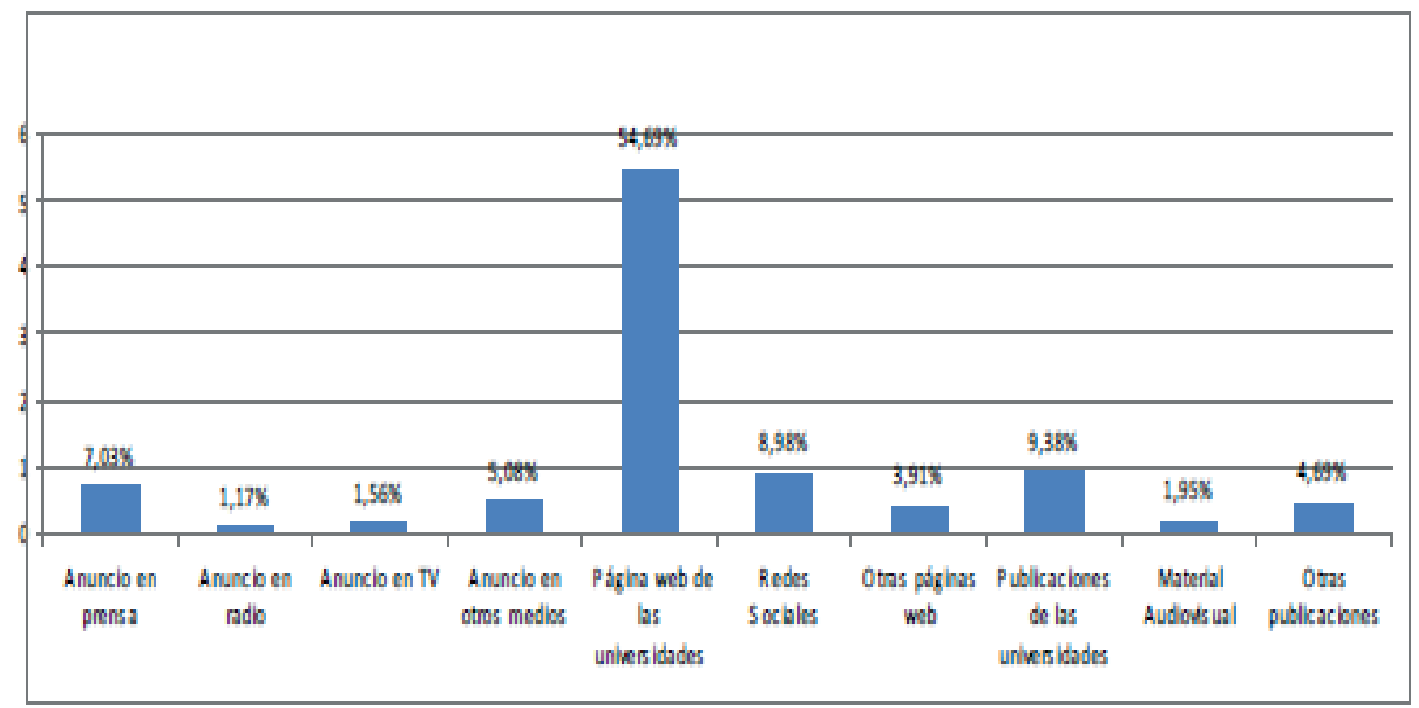

Figura 1. Porcentaje de medios de comunicación consultados como fuente de _. 
En la Figura 1 podemos observar que las publicaciones universitarias conservan unas índices de consulta significativos, susceptibles de aumentar gracias a consultas de otras publicaciones. Los anuncios tradicionales, afectados por la dinámica económica general de los últimos años, presentan unas cifras modestas. La prensa aventaja a la televisión y a la radio, fuentes periclitadas a la hora de atraer a los futuros estudiantes universitarios en el momento de informarse sobre la oferta de educación superior.

Como los anuncios en otros medios incluyen a los soportes digitales, Internet y la web 2.0 refuerzan su posición predominante, a la que se pueden añadir otras páginas web consultadas $(3,91 \%$ de la muestra). En total, el 72,66\% (el sumatorio de páginas web de las Universidades, redes sociales, otras páginas web y anuncios en otros medios digitales) de las consultas proceden del universo web 2.0.

Las páginas web de las Universidades, cuya supremacía es arrolladora, actúan como retro alimentadoras del círculo virtuoso que envuelve a Internet. El hecho que el $54,69 \%$ de encuestados consulten los sitios web demuestra la necesidad de este escaparate virtual, no tan sólo en el sector de la enseñanza, sino en el resto de sectores. Una Universidad sin un web bien pertrechado puede dilapidar opciones de atracción de estudiantes. La usabilidad y la navegabilidad de la página pueden condicionar la elección, en un entorno de rapidez y exigencia máxima por parte de las generaciones de nativos digitales. Un tiempo de descarga excesivo en la página actúa como freno en muchas ocasiones.

En un posible engagement página web de la Universidad-redes sociales ubicamos la convergencia tecnológica y el contexto multiplataforma del IMS (IP Multimedia Subsystem) o IMP (IP Multimedia Platform). La generación de algoritmos que crucen datos sobre perfiles de los usuarios, tipos de carreras a elegir, lugares de procedencia y otras variables significativas puede dar lugar a programas de gestión eficiente o a una suerte de CRM (Customer Relationship Management) que automatice la toma de decisión y envíe contenidos adaptados al usuario.

La customización es sencilla utilizando esas herramientas. En este campo, el desarrollo de las UC (Unified Communications) presenta perspectivas de desarrollo cuyas implicaciones en términos de ROI (Retorno Operativo de la Inversión) pueden ser determinantes a corto plazo. Como el público objetivo está formado por jóvenes habituados a la rapidez y al uso de las redes, para la Universidad es relevante exprimir el factor de automatismo. Esa interpretación ya ha alcanzado a los medios de comunicación, que permiten reenviar contenidos a través de estas redes (Figura 2). 
Hay que tener en cuenta que la navegación en redes rompe las teóricas barreras de Internet (saturación, desorientación o falta de jerarquización) y potencia la ruptura de los límites espaciales, temporales y de la secuencia, además de propulsar la visualidad, la interactividad, la hipertextualidad y el multimedia (Fondevila Gascón, 2010).

Es muy probable, en este entorno, que proliferen las interacciones entre las páginas web y las redes sociales. Es por ello que en la UAO se está estudiando el impacto de las redes sociales en el SEM (Social Engine Marketing), el SEO (Social Engine Optimization) y, sobre todo, el SMM (Social Media Marketing), que genera nuevos yacimientos de ocupación como las figuras del community manager o community recorder.

Tabla 1. Uso de las redes sociales por parte de los medios de comunicación. Fuente: Elaboración propia

\begin{tabular}{|c|c|c|c|c|}
\hline & \multicolumn{4}{|c|}{ Medio de comunicación } \\
\hline $\begin{array}{c}\text { Red } \\
\text { social }\end{array}$ & E1 Минđо & La Vanguardia & $\begin{array}{c}\text { Financial } \\
\text { Times }\end{array}$ & $\begin{array}{c}\text { The Wall Street } \\
\text { Journal }\end{array}$ \\
\hline Youtube & NO & $\overline{\text { sí }}$ & Sí & Sí \\
\hline LinkedIn & Sí & sí & Sí & Sí \\
\hline Delicious & NO & No & NO & NO \\
\hline FlickR & NO & NO & Sí & NO \\
\hline $\mathrm{Hi5}$ & NO & NO & NO & sí \\
\hline MySpace & NO & NO & NO & NO \\
\hline
\end{tabular}

\begin{tabular}{|c|c|c|c|c|}
\hline Facebook & sí & sí & sí & sí \\
\hline Twitter & sí & sí & sí & sí \\
\hline
\end{tabular}


Los anuncios en prensa se sitúan cerca del binomio de vanguardia (16\%), mientras que el resto de fuentes se ubican a continuación, pero a distancia. El crecimiento en la penetración y uso de Internet y de las redes sociales hace prever que éstas logren la primera plaza a corto plazo.

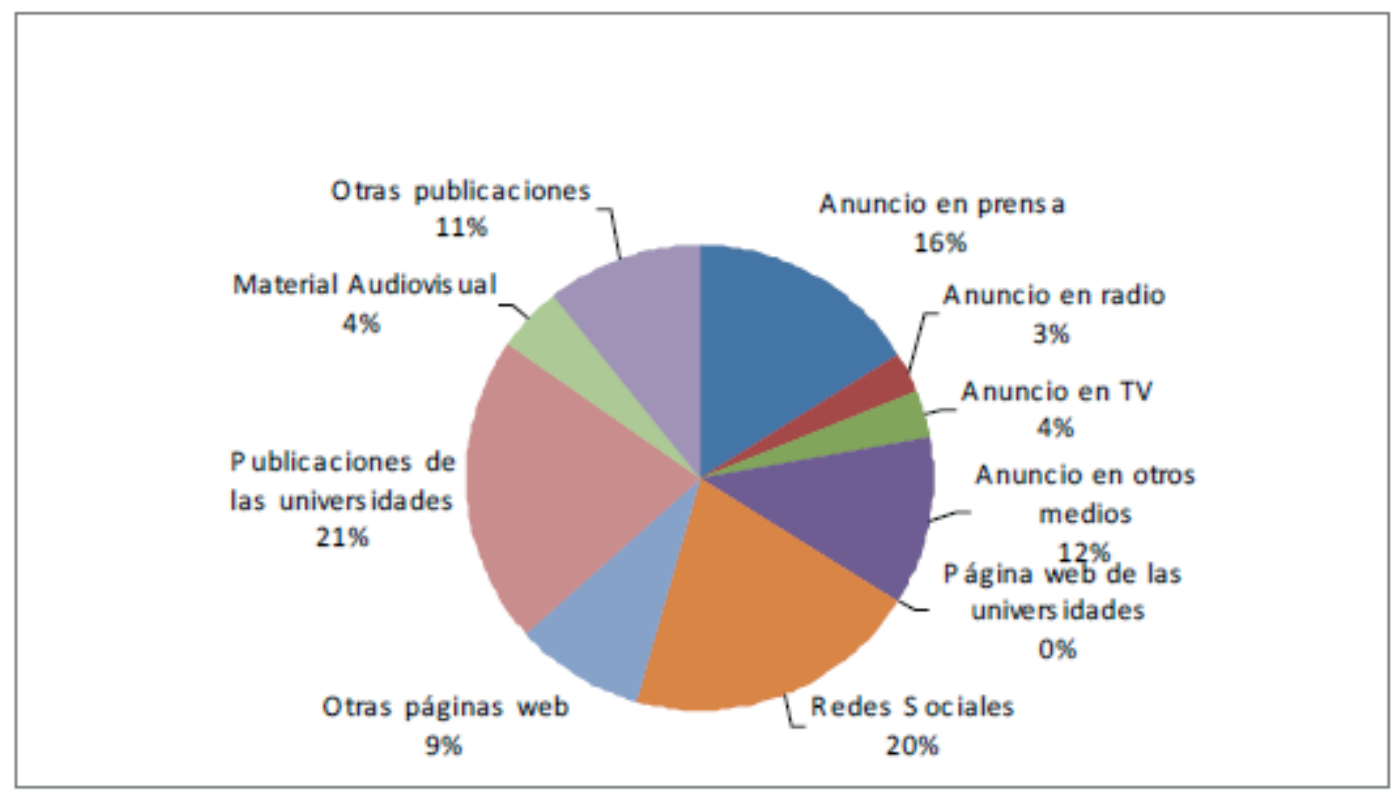

Figura 2. Porcentaje de medios de comunicación consultados como fuente de información sobre las Universidades sin contar las páginas web de aquéllas. Fuente: Elaboración propia

Otro foco de análisis es la distribución de consultas según el género (Figura 3). En el caso de las páginas web de las Universidades, la mujer las consulta más que el hombre (un elevado $15,65 \%$ de diferencia). Cuando el ítem analizado son las redes sociales, los hombres acuden a ellas $(11,70 \%)$ en una cantidad que se sitúa por encima de las mujeres $(7,59 \%$ ), un 4,11\% más en números absolutos (un 35,13\% más en cifras relativas dentro de las redes sociales). La consulta de otras páginas web está encabezada por los hombres. Una diferencia llamativa se produce en la consulta de anuncios en otros medios digitales (9,57\% de hombres, $2,53 \%$ de mujeres).

Si sumamos las cuatro fuentes que se pueden incluir en el entorno web 2.0, el porcentaje de consultas es de un $74,04 \%$ por parte de las mujeres y de un $73,39 \%$ por parte de los hombres. La diferencia es insignificante, y la variación se registra en la distribución interna de las fuentes digitales consultadas. 


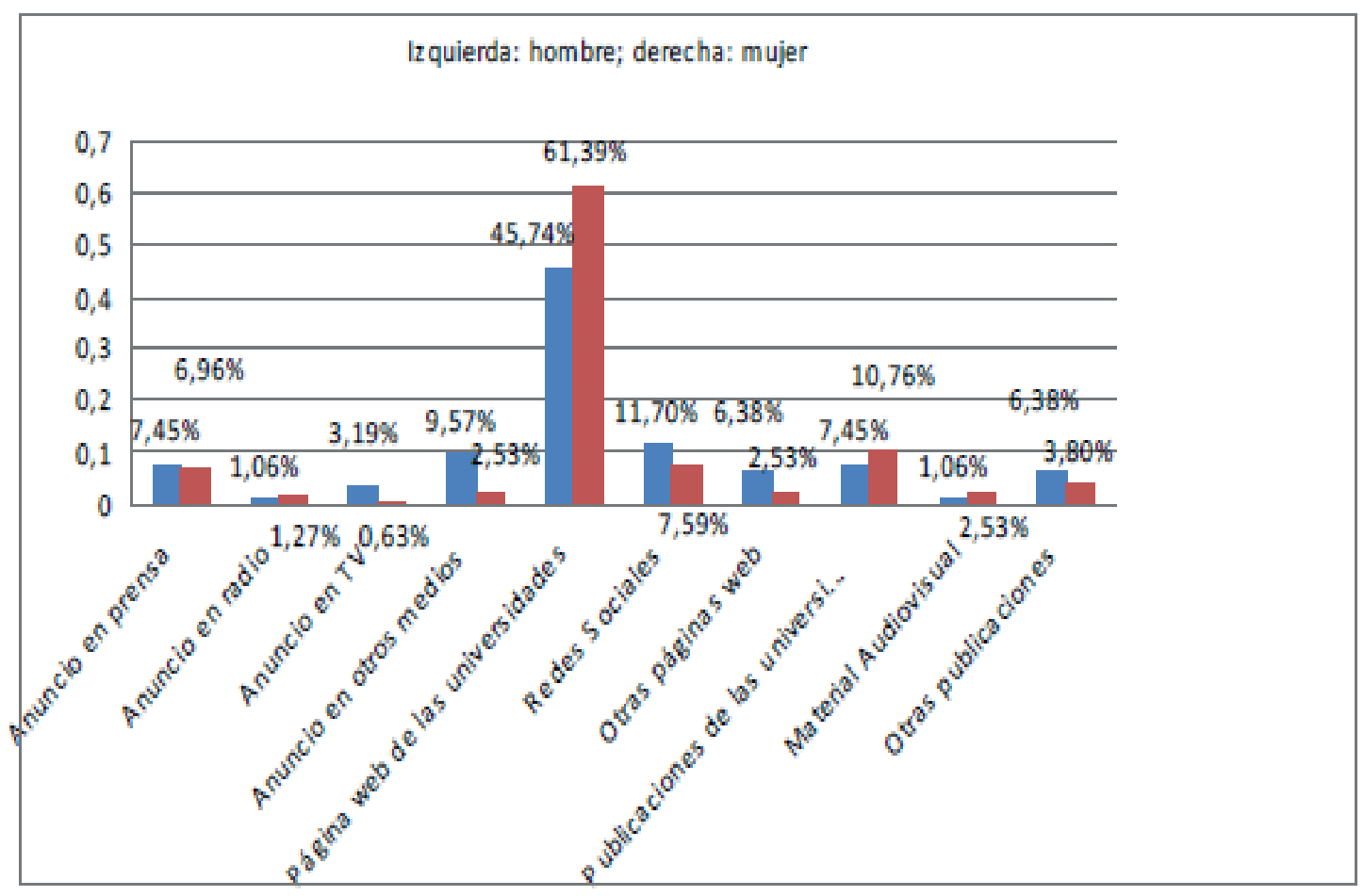

Figura 3. Porcentaje de medios de comunicación consultados como fuente de información sobre las Universidades según el género. Fuente: Elaboración propia

A tenor de los datos empíricos, las redes sociales e Internet se erigen en la principal fuente de información de los estudiantes que pretenden acceder a la Universidad.

Ese resultado está en la línea de datos de 2010 (Ontsi, 2010) según los cuales los particulares que habían utilizado Internet para buscar datos sobre formación y educación eran un 52,5\% y los que habían consultado Internet para algún tipo de aprendizaje, el 45,8\%. La dinámica virtual, como se puede comprobar, extiende sus tentáculos de manera holística e irreversible.

\section{CONCLUSIÓN}

Las redes sociales se están configurando como un canal creciente de búsqueda de información, arañando porcentajes a medios de comunicación tradicionales y demostrando la tendencia imparable del universo 2.0. A esa dinámica no escapa la Universidad. En esta investigación se ha demostrado que las redes sociales son una fuente de información relevante en el momento de tomar la decisión de elegir Universidad. Así, las redes sociales rozan el $9 \%$ de consultas y ocupan la tercera posición como fuente. 
Sólo las páginas web de las Universidades y las publicaciones de las Universidades cuentan con más adeptos a la hora de informarse, aunque las páginas web se hallan también en la dinámica virtual que caracteriza, cada vez más, a las generaciones de nativos digitales. En esa línea, las fuentes que podemos englobar en el ecosistema web 2.0 (páginas web de las Universidades, redes sociales, otras páginas web y anuncios en otros medios digitales) suman un $72,66 \%$ de consultas, una cifra muy elevada que puede modificar las estrategias de marketing de las instituciones de enseñanza superior. En un análisis por géneros, el masculino consulta más las redes sociales, las otras páginas web y los anuncios en otros medios digitales, mientras que el femenino opta en mayor medida por las páginas web de las Universidades.

Como la tendencia del mercado conduce a más inserciones en soportes digitales en detrimento de las tradicionales, las Universidades deben reorientar sus políticas de comunicación hacia Internet, redes sociales y herramientas del web 2.0. Se trata de fuentes de contenido al alza, que se están convirtiendo en un referente en el proceso de búsqueda de información y que requerirán de un seguimiento que permita llevar a cabo estudios longitudinales para confirmar el alcance del fenómeno.

\section{REFERENCIAS}

Crozier, G. (1997). Empowering the powerful: a discussion of the interrelation of government policies and consumerism with social class factors and the impact of this upon parent interventions in their children's schooling. British Journal of Sociology of Education, 18(2).

Fita, E.; Rodríguez, S. \& Torrado, M. (2004). El rendimiento académico en la transición secundaria-universidad. Revista de educación, 334: 391-414

Fondevila Gascón, J. F. (2008). La alianza entre el periodismo digital y tradicional y los operadores de telecomunicación: hacia un rendimiento óptimo de la red. Trabajo presentado al II Congreso Nacional Ulepicc-España. Barcelona.

Fondevila Gascón, J. F. (2010). Visual Impact in the Digital Press: Spanish Empirical Research. Brazilian Journalism Research, 6 (2): 120- 137.

Fondevila Gascón, J. F. (2011). Cable and Broadband in Spain, 2010. Terrassa: CECABLE.

Fondevila Gascón, J. F. \& Carreras Alcalde, M. (2010). La tecnologia com a eina al servei de l'educació: una visió humanitzadora. Trabajo presentado al Congreso 
Frotuny, M.; Pujol, M. R. \& Borja Solé, M. (1991). Estudio diacrónico sobre la elección de las carreras superiores en función del sexo: propuestas de algunas medidas para conseguir la igualdad de oportunidades. Bordón. Revista de pedagogía, 43(1): 91-104.

Gilder, G. (2002). Telecosm: The World After Bandwidth Abundance. New York: Touchstone.

Mosterio, M. J. \& Porto, A. M. (2000). Los motivos de elección de estudios en alumnos y alumnas de universidad. Innovación educativa, 10: 121-132.

ONTSI. (2010). Indicadores de seguimiento de la Sociedad de la Información en España. Madrid: Ontsi.

ONTSI. (2011): Indicadores destacados de la Sociedad de la Información en España. Madrid: Ontsi.

Pampaloni, A. M. (2010). The influence of organizational image on college selection: what students seek in institutions of higher education. Journal of Marketing for Higher Education, 20(1): 19-48.

Roszkowski, M. J. \& Spreat, S. (2010). Weighing the difference: the validity of multiplicative and subtractive approaches to item weights in an instrument assessing college choice decisions. Journal of Marketing for Higher Education, 20(2): 209-239.

THE COCKTAIL ANALYSIS. (2010). Segunda Oleada del Observatorio de Redes Sociales. Madrid: The Cocktail Analysis.

Veleda, C. (2002). Estrategias individuales y familiares de elección de instituciones de educación superior. Buenos Aires: IIPE-UNESCO.

West, A.; Varlaam, A. \& Scott, G. (1991). Choice of high schools: pupils perceptions. Educational Research, 33 (3): 205-215. 


\section{Joan Francesc Fondevila Gascón}

Doctor en Periodismo por la Universitat Autònoma de Barcelona (UAB), Licenciado en Ciencias de la Información, Licenciado en Ciencias Políticas y Sociología, Máster en Periodismo y Ciencias de la Comunicación y Máster en Comunicación y Deporte. Profesor en la Universitat Abat Oliba CEU (UAO) de Barcelona, profesor del Doctorado en Sociedad de la Información y el Conocimiento y del Máster Universitario de Sociedad de la Información y el Conocimiento de la Universitat Oberta de Catalunya (UOC), y profesor de la Universitat Autònoma de Barcelona y la Universitat Politècnica de Catalunya (UPC). Es fundador y director del Centro de Estudios sobre el Cable (CECABLE).

\section{José Luis del Olmo Arriaga}

Doctor en Comercialización e Investigación de Mercados. Licenciado en Ciencias de la Información por Universitat Autònoma de Barcelona (UAB). Profesor de Dirección Comercial en la Universitat Abat Oliba CEU, especializado en Marketing sectorial.

\section{Javier Sierra Sánchez}

Doctor en Ciencias de la Información y profesor de Radio en la Universitat Abat Oliba CEU. 